\title{
Duality of planar and spacial curves: new insight
}

\author{
Viktor S. Kulikov ${ }^{1}$ • Eugenii Shustin ${ }^{2}$
}

Received: 23 December 2014 / Accepted: 12 May 2015 / Published online: 5 August 2015

(C) Springer International Publishing AG 2015

\begin{abstract}
We study the geometry of equiclassical strata of the discriminant in the space of plane curves of a given degree, which are families of curves of given degree, genus and class (degree of the dual curve). Our main observation is that the use of duality transformation leads to a series of new sufficient conditions for a regular behavior of the equiclassical stratification. We also discuss duality of curves in higherdimensional projective spaces and in Grassmannians with focus on similar questions of the regularity of equiclassical families of spacial curves.
\end{abstract}

Keywords Planar curves - Spacial curves · Duality of curves · Equiclassical stratification

Mathematics Subject Classification $14 \mathrm{H} 10 \cdot 14 \mathrm{H} 20 \cdot 14 \mathrm{H} 50$

\footnotetext{
The first author has been supported by Grants of RFBR 14-01-00160 and 15-01-02158, and by the Government of the Russian Federation within the framework of the implementation of the 5-100 Programme Roadmap of the National Research University Higher School of Economics, AG Laboratory. The second author has been supported by the Hermann-Minkowski-Minerva Center for Geometry at the Tel Aviv University and by the German-Israeli Foundation Grant No. 1174-197.6/2011.
}

Viktor S. Kulikov

kulikov@mi.ras.ru

Eugenii Shustin

shustin@post.tau.ac.il

1 Steklov Mathematical Institute, 8 Gubkina Str., 119991 Moscow, Russia

2 School of Mathematical Sciences, Raymond and Beverly Sackler Faculty of Exact Sciences, Tel Aviv University, Ramat Aviv, 69978 Tel Aviv, Israel 


\section{Introduction}

Our main goal is to show that the issue of duality of planar and spacial projective curves is not exhausted yet, and it still yields new results and raises interesting open problems.

The duality of plane algebraic curves is a classically known relation, which has been used in the study of plane algebraic curves since the nineteenth century. It is tightly linked to the stratification of the discriminant in the space of curves of a given degree into equiclassical strata, i.e. families of curves of given degree, genus and class (degree of the dual curve). However, previous considerations of the geometry of equiclassical strata [9,29] have not taken the duality relation into account. We address regularity properties of equiclassical strata, like that to be locally transverse intersection, to be irreducible, to have a nodal-cuspidal general member, and we show that a combination of duality isomorphisms with tools of deformation theory leads to a series of new numerical sufficient conditions for the above regularity properties. The results of our study are absorbed in Theorems 2.5, 2.9, 2.11, 2.15, and 2.17 in Sect. 2. For example, by [9, Theorem 1.2] and [29, Theorem 1.1], the equiclassical family $V_{d, g, c}$ parameterizing irreducible curves of degree $d$, genus $g$, and class $c$ has expected dimension $d-g+c+1$ and its generic member is a curve with ordinary nodes and cusps if $\varkappa-2 \delta \leq 3 d-4$, where $\varkappa=d(d-1)-c, 2 \delta=(d-1)(d-2)-2 g$. We show that $V_{d, g, c}$ possesses the above properties as long as $5 \varkappa-6 \delta \leq d^{2}+6 d-3$, cf. Theorem 2.15 (b) in Sect. 2.

The duality between non-degenerate curves in higher-dimensional spaces was discovered by Piene [24, Section 5], and later extended to curves in Grassmannians [23]. In Sect. 3, we provide a brief account of this duality relation with elementary proofs, introduce equiclassical families of spacial curves and formulate several open questions on their geometry and on duality morphisms between them.

We work over the complex field, though most of results can be stated over any algebraically closed field of characteristic zero.

\section{Equiclassical families of plane curves and duality}

The discriminant $\Delta_{d}$ in the linear system $\left|\mathcal{O}_{\mathbb{P}^{2}}(d)\right|$ of plane curves of a given degree $d$ admits natural stratifications into families, parameterizing singular curves with specific properties. In particular, the part $\Delta_{d}^{\text {irr }} \subset \Delta_{d}$ formed by reduced, irreducible curves splits into the disjoint union of Severi varieties $V_{d, g}$ that parameterize curves of degree $d$ and genus $g$. These are the most important strata of the discriminant, since they are tightly related to the Gromov-Witten theory and geometry of moduli spaces of curves. Severi varieties are well-studied: in particular, for any $d$ and $g$ satisfying

$$
0 \leq g \leq \frac{(d-1)(d-2)}{2}
$$

$V_{d, g}$ is a non-empty quasiprojective irreducible variety of dimension $3 d+g-1[18,27]$; furthermore, a generic member of $V_{d, g}$ is a nodal curve [2,18,32] (a similar statement for other rational surfaces can be found in [5]). More thorough stratification consists 
of equisingular families of curves that parameterize curves of a given degree having singular points of prescribed topological or analytic types. Except for small degrees or for curves with only ordinary nodes, no complete description of such a stratification is known, even the non-emptiness question on families of curves with ordinary nodes and cusps is not completely answered (we refer the reader to [13] for a survey and references on this subject). In the present paper, we address the regularity properties of the equiclassical stratification of $\Delta_{d}^{\mathrm{irr}}$, which is intermediate between the Severi and the equisingular stratification. It consists of the equiclassical families $V_{d, g, c}$ that parameterize curves of degree $d$, genus $g$, and class $c$ (degree of the dual curve). A remarkable property of the equiclassical stratification is a series of coupling duality isomorphisms.

Lemma 2.1 The duality transformation of plane curves defines isomorphisms

$$
\mathcal{D}_{d, g, c}: V_{d, g, c} \stackrel{\simeq}{\longrightarrow} V_{c, g, d}, \quad \mathcal{D}_{c, g, d}: V_{c, g, d} \stackrel{\simeq}{\longrightarrow} V_{d, g, c}
$$

for all $d, c \geq 2, g \geq 0$.

Proof We only explain that $\mathcal{D}_{d, g, c}$ and $\mathcal{D}_{c, g, d}$ are morphisms between quasiprojective varieties. Let $U_{d} \subset\left|\mathcal{O}_{\mathbb{P}^{2}}(d)\right|$ be the set of smooth curves of degree $d$. The duality transformation $\mathcal{D}_{d}: U_{d} \rightarrow\left|\mathcal{O}_{\mathbb{P}^{2}}(d(d-1))\right|$ is a well-defined morphism, which can explicitly be written as a homogeneous polynomial map in coefficients of curve equations. The same polynomial formulas correctly extend to $U_{d} \cup \Delta^{\text {irr }}$ so that, for each curve $C \in V_{d, g, c} \subset \Delta^{\text {irr }}$ with $c<d(d-1)$, the image $\mathcal{D}_{d}(C)$ is a reducible curve containing a component $C^{\prime}=\mathcal{D}_{d, g, c}(C) \in V_{c, g, d}$ and $d(d-1)-c$ lines (these lines are multiple tangents to $C^{\prime}$, counted with appropriate multiplicities). Hence, $\mathcal{D}_{d}$ restricts to $V_{d, g, c}$ as a well-defined morphism $\mathcal{D}_{d, g, c}: V_{d, g, c} \rightarrow V_{c, g, d}$.

Equiclassical families have been studied by Diaz and Harris [9]. Answering a question by Fulton, they proved

Theorem 2.2 ([9, Theorem 1.2]) If $V_{d, g, c} \neq \emptyset$ and

$$
c \geq 2 g-1
$$

then a generic member of any component of $V_{d, g, c}$ is a curve with nodes and cusps.

Later on it was slightly improved by the second author as follows.

Theorem 2.3 ([29, Theorem 1.1]) If $V_{d, g, c} \neq \varnothing$ and

$$
c \geq 2 g-d+2
$$

then a generic member of any component of $V_{d, g, c}$ is a curve with nodes and cusps.

It is not difficult to show, see [9,29] or Theorem 2.9 below, that under either of conditions (2) or (3), $V_{d, g, c}$ is equidimensional of (expected) dimension

$$
\operatorname{dim} V_{d, g, c}=\operatorname{dim}_{\exp } V_{d, g, c}=d-g+c+1 .
$$


Our aim is to study geometry of equiclassical families in more detail. We will give a series of sufficient numerical conditions for the non-emptiness, expected dimension, irreducibility, and the nodal-cuspidal generic member of (any component of) an equiclassical family $V_{d, g, c}$, as well as for a regular adjacency of equiclassical strata:

- in Sect. 2.2 we mainly revise known results (including those in $[9,20,21,29]$ ) and produce "linear" conditions, where the number of virtual (in the sense of [22]) cusps is at most linear in the degree,

- in Sect. 2.3, using methods of [12,28], we derive "quadratic" conditions, where the number of (virtual) cusps is bounded from above by a quadratic function of the degree,

- furthermore, we intertwine both, linear and quadratic conditions with the duality isomorphisms (1), which results in additional regularity conditions, not covered by any other criterion.

\subsection{Local structure of equiclassical families}

Let $C \in V_{d, g, c}, z \in \operatorname{Sing}(C)$. The $\delta$ - and $\varkappa$-invariants of the germ $(C, z)$ are, see [9, Definition 3.12] or [14, Section I.3.4] for details:

$$
\varkappa(C, z)=\left(C \cdot C_{p}\right)_{z}, \quad \delta(C, z)=\frac{1}{2}(\varkappa(C, z)-\operatorname{mt}(C, z)+r(C, z)),
$$

where $C_{p}$ denotes a generic polar of $C,(* * *)_{z}$ the intersection number at the point $z, \operatorname{mt}(C, z)=(C \cdot L)_{z}$ the multiplicity of the singular point $z$ of $C, L$ being a generic straight line passing through $z$, and, finally, $r(C, z)$ denotes the number of irreducible components of the germ $(C, z)$.

Furthermore, given a (local) equation $f(x, y)=0$ of the germ $(C, z)$, we identify the semiuniversal deformation base $\mathrm{B}_{z}$ of the germ $(C, z)$ with $\mathcal{O}_{\mathbb{P}^{2}, z} / J(C, z)$, where $J(C, z)=\langle f, \partial f / \partial x, \partial f / \partial y\rangle \subset \mathcal{O}_{\mathbb{P}^{2}, z}$ is the Tjurina ideal, cf. [9, Section 5]. By [8] and [9, Definition 4.3 and Theorem 5.5], the equiclassical locus $\mathrm{EC}_{z} \subset \mathrm{B}_{z}$ (which parameterizes $\delta$ - and $\varkappa$-constant deformations) is an irreducible analytic variety germ, which has codimension $\varkappa(C, z)-\delta(C, z)$ with the tangent cone supported at the linear space $J^{\mathrm{ec}}(C, z) / J(C, z)$, where $J^{\mathrm{ec}}(C, z) \subset \mathcal{O}_{C, z}$ is the ideal defined by

$$
J^{\mathrm{ec}}(C, z)=\left\{\varphi \in \mathcal{O}_{\mathbb{P}^{2}, z}: \begin{array}{l}
\text { ord }\left.\varphi\right|_{P} \geq\left(C_{p} \cdot P\right) \text { for each } \\
\text { irreducible component } P \subset(C, z)
\end{array}\right\} .
$$

We have a natural map

$$
\Phi_{C}:\left(\left|\mathcal{O}_{\mathbb{P}^{2}}(d)\right|, C\right) \rightarrow \prod_{z \in \operatorname{Sing}(C)} \mathrm{B}_{z},
$$

and the germ of $V_{d, g, c}$ at $C$ can be viewed as

$$
\left(V_{d, g, c}, C\right)=\Phi^{-1}\left(\prod_{z \in \operatorname{Sing}(C)} \mathrm{EC}_{z}\right)
$$


Notice that $\mathrm{EC}_{z}$ and hence $V_{d, g, c}$ may not be smooth, see [8, Theorem 28 and Lemma 29]. We, however, can speak about the following property.

Definition 2.4 We say that the germ of the family $V_{d, g, c}$ at $C \in V_{d, g, c}$ is a locally transverse intersection (shortly LTI), if $\operatorname{Im} \Phi_{C}$ intersects transversally with $\prod_{z \in \operatorname{Sing}(C)} \mathrm{EC}_{z}$ in $\prod_{z \in \operatorname{Sing}(C)} \mathrm{B}_{z}$. Respectively, $V_{d, g, c}$ is called LTI, if all germs $\left(V_{d, g, c}, C\right)$ are LTI for all $C \in V_{d, g, c}$.

It is evident that the duality isomorphisms (1) preserve the expected dimension of equiclassical families, cf. formula (4). We can say even more.

Theorem 2.5 (a) Let $V_{d, g, c}$ be LTI. Then $V_{d, g, c}$ has expected dimension $c-g+d+1$, and satisfies the following incidence relations:

$$
\begin{aligned}
& V_{d, g, c} \subset \bar{V}_{d, g, c+1} \text {, if } 2 d-2-c+2 g \\
& =\sum_{z \in \operatorname{Sing}(C)}(\kappa(C, z)-2 \delta(C, z))>0, \\
& V_{d, g, c} \subset \bar{V}_{d, g+1, c+2} \text {, if } c-3 g+\left(d^{2}-7 d+6\right) / 2 \\
& =\sum_{z \in \operatorname{Sing}(C, z)}(3 \delta(C, z)-\kappa(C, z))>0,
\end{aligned}
$$

$C$ being any curve in $V_{d, g, c}$.

(b) The family $V_{d, g, c}$ is LTI if and only if the family $V_{c, g, d}$ is LTI.

Remark 2.6 Note that the first inclusion in (6) means deforming a cusp to a node, while the second inclusion means smoothing out a node of a generic curve in $V_{d, g, c}$.

Proof (a) For the first statement, we notice that the LTI property yields

$$
\operatorname{dim} V_{d, g, c}=\frac{d(d+3)}{2}-\sum_{z \in \operatorname{Sing}(C)}(\varkappa(C, z)-\delta(C, z))=d-g+c+1,
$$

where the latter equality follows from the Plücker and genus formulas

$$
c=d(d-1)-\varkappa(C), \quad g=\frac{(d-1)(d-2)}{2}-\delta(C),
$$

where

$$
\varkappa(C)=\sum_{z \in \operatorname{Sing}(C)} \varkappa(C, z), \quad \delta(C)=\sum_{z \in \operatorname{Sing}(C)} \delta(C, z) .
$$

Now pick $C \in V_{d, g, c}$ and show that $C \in \bar{V}_{d, g, c+1}$ or $C \in \bar{V}_{d, g+1, c+2}$ accordingly as $2 d-2-c+2 g>0$ or $c-3 g-\left(d^{2}-7 d+6\right) / 2>0$. Since

$$
2 d-2-c+2 g=\varkappa-2 \delta, \quad c-3 g+\frac{d^{2}-7 d+6}{2}=3 \delta-\varkappa,
$$


we can interpret these numbers as follows. By [9, Theorem 1.1], a generic germ of an equiclassical locus $\mathrm{EC}_{z}$, where $z \in \operatorname{Sing}(C), C \in V_{d, g, c}$, has $\varkappa(C, z)-2 \delta(C, z)$ cusps and $3 \delta(C, z)-\varkappa(C, z)$ nodes as its only singularities. Hence, $\varkappa-2 \delta$ and $3 \delta-\varkappa$ are the total numbers of cusps, resp. nodes over generic members of $\mathrm{EC}_{z}, z \in$ $\operatorname{Sing}(C)$. If $x-2 \delta>0$, then there is $z \in \operatorname{Sing}(C)$ such that $\varkappa(C, z)-2 \delta(C, z)>0$, and hence $\mathrm{EC}_{z} \subset \overline{\mathrm{EC}}_{z}^{\prime}$, where $\mathrm{EC}_{z}^{\prime} \subset \mathrm{B}_{z}$ is the locus formed by the germs having $\varkappa(C, z)-2 \delta(C, z)-1$ cusps and $3 \delta(C, z)-\varkappa(C, z)+1$ nodes $^{1}$. It follows from the LTI property that $C \in \bar{V}_{d, g, c+1}$. If $3 \delta-\varkappa>0$, then there is $z \in \operatorname{Sing}(C)$ such that $3 \delta(C, z)-\varkappa(C, z)>0$, and hence $\mathrm{EC}_{z} \subset \overline{\mathrm{EC}}_{z}^{\prime \prime}$, where $\mathrm{EC}_{z}^{\prime \prime} \subset \mathrm{B}_{z}$ is the locus formed by the germs having $\varkappa(C, z)-2 \delta(C, z)$ cusps and $3 \delta(C, z)-\varkappa(C, z)-1$ nodes. Again the LTI property yields that $C \in \bar{V}_{d, g+1, c+2}$.

(b) For the second statement, for each point $z \in \operatorname{Sing}(C)$, introduce the zerodimensional scheme $Z_{C, z}^{\mathrm{ec}} \subset \mathbb{P}^{2}$ supported at $z$ and defined by the ideal $J^{\mathrm{ec}}(C, z)$. Set $Z^{\mathrm{ec}}(C)=\bigcup_{z \in \operatorname{Sing}(C)} Z_{C, z}^{\mathrm{ec}}$. By [9, Lemma 5.3], $\operatorname{deg} Z^{\mathrm{ec}}(C)=\varkappa-\delta$. The LTI property can equivalently be expressed as the $h^{1}$-vanishing

$$
H^{1}\left(\mathbb{P}^{2}, \partial_{Z^{\mathrm{ec}}(C)}(d)\right)=0
$$

where $\mathcal{J}_{Z^{\mathrm{ec}}(C)}$ is the ideal sheaf of the scheme $Z^{\mathrm{ec}}(C)$. We rewrite condition (7) as follows. Let $F\left(x_{0}, x_{1}, x_{2}\right)=0$ be a (homogeneous) equation of a curve $C \in V_{d, g, c}$. Choose a generic point $p=\left(p_{0}, p_{1}, p_{2}\right)$ and a generic line $L_{q}=q_{0} x_{0}+q_{1} x_{1}+q_{2} x_{2}=$ 0 in the plane so that

- the polar curve $C_{p}=\left\{p_{0} F_{x_{0}}+p_{1} F_{x_{1}}+p_{2} F_{x_{2}}=0\right\}$ intersects $C$ at each points $z_{i} \in \operatorname{Sing}(C)$ with multiplicity $\left(C_{p} \cdot C\right)_{z_{i}}=\varkappa\left(C, z_{i}\right), i=1, \ldots, r$, and in a set $S_{p} \subset C \backslash \operatorname{Sing}(C)$ with the total multiplicity $c$;

- the set $T_{q}=L_{q} \cap C$ is disjoint from $\operatorname{Sing}(C) \cup S_{p}$, and $\left(L_{q} \cdot C\right)_{T_{q}}=d$.

Then, cf. [9, Proof of Lemma 5.14], [29, Proof of Lemma 2.1],

$$
H^{1}\left(\mathbb{P}^{2}, \partial_{Z^{\mathrm{ec}}(C)}(d)\right)=H^{1}\left(\widehat{C}, \mathcal{O}_{\widehat{C}}\left(v^{*}\left(S_{p}\right)+v^{*}\left(T_{q}\right)\right)\right),
$$

where $v: \widehat{C} \rightarrow C$ is the normalization. Let $C^{\vee} \in V_{c, g, d}$ be the dual curve, $v^{\vee}: \widehat{C} \rightarrow C^{\vee}$ the dual normalization, and let $C^{\vee}$ be given by a homogeneous equation $F^{\vee}\left(y_{0}, y_{1}, y_{2}\right)=0$. Then the polar $C_{q}^{\vee}=\left\{q_{0} F_{y_{0}}^{\vee}+q_{1} F_{y_{1}}^{\vee}+q_{2} F_{y_{2}}^{\vee}=0\right\}$ intersects $C^{\vee}$ in $\operatorname{Sing}\left(C^{\vee}\right)$ and in a set $S_{q}^{\vee}$ dual to $T_{q} \subset C$, whereas the line $L_{p}^{\vee}=\left\{p_{0} y_{0}+p_{1} y_{1}+p_{2} y_{2}=0\right\}$ intersects $C^{\vee}$ in a set $T_{p}^{\vee} \subset C^{\vee} \backslash\left(\operatorname{Sing}\left(C^{\vee}\right) \cup S_{q}^{\vee}\right)$ dual to $S_{p} \subset C$. Hence $v^{*}\left(S_{p}\right)=\left(v^{\vee}\right)^{*}\left(T_{p}^{\vee}\right), v^{*}\left(T_{q}\right)=\left(v^{\vee}\right)^{*}\left(S_{q}^{\vee}\right)$, which completes the proof in view of

$$
H^{1}\left(\mathbb{P}^{2}, \mathcal{J}_{Z^{\mathrm{ec}}\left(C^{\vee}\right)}(c)\right)=H^{1}\left(\widehat{C}, \mathcal{O}_{\widehat{C}}\left(\left(v^{\vee}\right)^{*}\left(S_{q}^{\vee}\right)+\left(v^{\vee}\right)^{*}\left(T_{p}^{\vee}\right)\right)\right) .
$$

Remark 2.7 One can see from the proof of Theorem 2.5 that the values $2 d-2-c+2 g$ and $c-3 g+\left(d^{2}-7 d+6\right) / 2$ are always nonnegative, and their sum $\left(d^{2}-3 d+2\right) / 2-g$ is positive as long as $V_{d, g, c}$ contains singular curves.

\footnotetext{
1 Over a versal deformation base, all singularities can be independently deformed or preserved.
} 
Definition 2.8 We call a family $V_{d, g, c}$ locally regular if it is LTI, has expected dimension and satisfies the incidence relations (6).

A simple well-known example of locally regular families are Severi varieties $V_{d, g}$ (here $c=2 d-2+2 g$ ), see [27].

\subsection{Linear regularity conditions}

In the following theorem we present some elementary or known regularity conditions.

Theorem 2.9 (a) The family $V_{d, g, c}, d, c \geq 2$, is non-empty, if

$$
c \geq 2 g+\left[\frac{d+1}{2}\right]+1 \quad\left(\text { equivalently } \kappa-2 \delta \leq d+\left[\frac{d}{2}\right]-3\right) .
$$

(b) Let $V_{d, g, c} \neq \emptyset, d, c \geq 2$.

(b) If

$$
c-2 g+d \geq-1 \quad \text { (equivalently } \kappa-2 \delta \leq 3 d-1 \text { ) }
$$

then $V_{d, g, c}$ is locally regular.

( $\left.\mathrm{b}_{2}\right)$ If

$$
c-2 g+d \geq 2 \text { (equivalently } \kappa-2 \delta \leq 3 d-4 \text { ), }
$$

then a generic member of any component of $V_{d, g, c}$ is a curve with nodes and cusps.

(b3) If either

$$
\begin{array}{ll}
c \geq 2 g+2 d-5 & \text { (equivalently } \kappa-2 \delta \leq 3), \\
\text { or } c \geq 3 g+\frac{3 d-5}{2} \quad & \left(\text { equivalently } 3 \delta-\kappa \geq \frac{d^{2}-4 d+1}{2}\right), \\
\text { or } c \geq g+\frac{d^{2}-2 d-1}{2} & \left(\text { equivalently } \kappa-\delta \leq \frac{3 d-1}{2}\right)
\end{array}
$$

then $V_{d, g, c}$ is irreducible.

Proof Under condition (9), we will construct curves with $n$ nodes and $k$ cusps realizing the required values of $d, g, c$. We can rewrite (9) as $k \leq[3(d-2) / 2]$. It is classically known, see [25-27], and also [11, Corollary 6.3] for a modern treatment, that if $k<3 d$, then one can smooth out prescribed nodes and cusps of such a nodal-cuspidal curve and deform prescribed cusps into nodes. Hence, it is sufficient to construct the following nodal-cuspidal curves: a rational curve of degree $d=2 s$ with $k=3 s-3$ and a rational curve of degree $d=2 s+1$ with $k=3 s-2$. By Plücker formulas, the first curve is dual to a nodal rational curve of degree $c=s+1$, whereas the second curve is dual to a rational curve of degree $c=s+2$ having one cusp and $\left(s^{2}+s-2\right) / 2$ nodes. The existence of the latter curve is left to the reader as an elementary exercise. 
Claim $\left(b_{2}\right)$ is a copy of Theorem 2.3 , included here for completeness and comparison with other criteria. Claim $\left(b_{1}\right)$ (which basically coincides with [9, Corollary 5.2]) holds, since (10) implies (7): indeed, in view of (8) it is enough to show that

$$
2 g-2<\operatorname{deg}\left(v^{*}\left(S_{p}\right)+v^{*}\left(T_{q}\right)\right)=c+d,
$$

which is equivalent to (10). In claim $\left(\mathrm{b}_{3}\right)$ we can suppose that $d, c \geq 3$, and then each inequality in (12) yields (3): it is evident for the first two inequalities, and the third one combined with $g \leq 2 g-d+1$ negative to (3) results in the impossible relation $g \geq\left(d^{2}-1\right) / 2$. Hence, by Theorem 2.3 , we are left with the irreducibility problem for families $V_{d}\left(n A_{1}, k A_{2}\right)$ of irreducible curves of degree $d$ with $n$ nodes and $k$ cusps. Each of the following conditions is sufficient for the irreducibility of $V_{d}\left(n A_{1}, k A_{2}\right)$ :

- $k \leq 3$, see [20], the missing cases of $k=3$ and $d=5$ or 6 are covered in [1, Theorem 2.4], [6], [7, Section II.7.3];

- $k \leq(d+1) / 2-g$, see $[21]$;

- $2 n+4 k<3 d$, see [15, Corollary IV.6.2].

In terms of $d, g$, and $c$ they specialize to the form (12).

Remark 2.10 The bound (9) is optimal for rational curves: a larger number of cusps simply is not possible by Plücker formulas as one can see in the proof of Theorem 2.9.

Now we combine Theorem 2.9 with isomorphisms (1). Notice, first, that linear conditions remain linear as long as $k \leq 3 d$ : Indeed, it follows from Plücker formulas that $3 d-k=3 c-k^{\vee}$, where $k$, resp. $k^{\vee}$, is the number of (virtual) cusps of the original, resp. dual curve. Second, the dualization of claims $(a),\left(b_{1}\right)$, and $\left(b_{2}\right)$ in Theorem 2.9 does not give anything new, whereas the dual to claim $\left(b_{3}\right)$ is a new statement.

Theorem 2.11 (a) If $V_{d, g, c} \neq \varnothing$ and either

$$
d \geq 2 g+2 c-5, \quad \text { or } d \geq 3 g+\frac{3 c-5}{2}, \quad \text { or } d \geq g+\frac{c^{2}-2 c-1}{2}
$$

then $V_{d, g, c}$ is irreducible.

(b) A non-empty family $V_{d}\left(n A_{1}, k A_{2}\right)$ is irreducible if either

$$
\begin{aligned}
3 n+4 k & \geq \frac{3 d^{2}-6 d-3}{2}, \\
\text { or } \quad 4 n+5 k & \geq \frac{6 d^{2}-14 d+1}{3}, \\
\text { or } \quad n+k & \geq \frac{d^{2}-5 d+\left(d^{2}-d-2 n-3 k-1\right)^{2}}{2} .
\end{aligned}
$$


Proof Conditions (13) are obtained from (12) by exchange of $d$ and $c$. Furthermore, rewriting (13) in the form

$$
\begin{aligned}
& c-2 g+d \geq 3 c-5, \quad c-2 g+d \geq g+\frac{5 c-5}{2}, \\
& c-2 g+d \geq\left(\frac{c^{2}-3 c+2}{2}-g\right)+\frac{2 c-3}{2},
\end{aligned}
$$

resp., we see that each of the inequalities (13) yields (11), and hence we get irreducibility criteria for families of curves with nodes and cusps in claim (b): via Plücker formulas, inequalities (13) are converted into (14).

Example 2.12 To show that Theorem 2.11 indeed contains a new information as compared with Theorem 2.9, we consider the case of curves of degree $d=7$ (for $d \leq 6$ the equisingular stratification of the discriminant is completely described $[1,6,7])$. So, Theorem $2.9\left(\mathrm{~b}_{3}\right)$ implies that $V_{7}\left(n A_{1}, k A_{2}\right)$ is irreducible if either

$$
k \leq 3, \quad \text { or } \quad k=4, \quad n=11, \quad \text { or } \quad k=4, \quad n \leq 3 \text {. }
$$

In addition to these cases, Theorem 2.11 implies the irreducibility of $V_{7}\left(n A_{1}, k A_{2}\right)$ for

$$
(k, n)=(6,9),(7,8), \text { and }(10,4)
$$

All these families are non-empty. One can easily find similar examples in higher degrees.

\subsection{Quadratic regularity conditions}

To formulate conditions, where the number of (virtual) cusps may depend quadratically on the degree, we replace the parameters $d, g, c$ by $d, \delta, \varkappa$ and use the notation $V_{d}^{\delta, \varkappa}=$ $V_{d, g, c}$, where $\varkappa=d(d-1)-c, \delta=(d-1)(d-2) / 2-g$ (that is $\delta=\delta(C)$ and $\varkappa=\varkappa(C)$ for any curve $\left.C \in V_{d}^{\delta, \varkappa}=V_{d, g, c}\right)$. For example, in terms of $\varkappa$ and $\delta$, condition (2), resp. (3), reads

$$
\varkappa-2 \delta \leq 2 d-1, \quad \text { resp. } \quad \varkappa-2 \delta \leq 3 d-4 .
$$

Due to the irregular behavior of families of plane curves with nodes and cusps (for instance, it is not known how many cusps a curve of degree $d \geq 9$ may have, cf. [3]), it is not possible to completely describe non-empty equiclassical families. Here we present the following partial result.

Theorem 2.13 Let $d \geq 3, \varkappa, \delta>0$.

(a) If $V_{d}^{\delta, \varkappa} \neq \emptyset$, then

$$
2 \delta \leq \varkappa \leq 3 \delta, \quad \delta \leq \frac{(d-1)(d-2)}{2}
$$


(b) If $d \leq 4$, then (15) is sufficient for the non-emptiness of $V_{d}^{\delta, \varkappa}$. If $d \geq 5$, then the conditions (15) and

$$
\varkappa-\delta \leq \frac{d^{2}-4 d+6}{2}
$$

are sufficient for the non-emptiness of $V_{d}^{\delta, \varkappa}$.

Proof (a) The first inequality in (15) follows from the second relation in (5) and from the relation $\delta(C, z) \geq \operatorname{mt}(C, z)(\mathrm{mt}(C, z)-1) / 2$, which follows from (5) too.

(b) By [29, Corollary 1.3], for $d \leq 10$ a generic member of any component of a nonempty family $V_{d}^{\delta, \varkappa}$ is a curve with nodes and cusps. Thus, the case of $d \leq 4$ reduces to the study of curves with nodes and cusps, which are classically known. For $d \geq 5$ we apply [28, Theorem 4.1] (suitably specified in [15, Theorem IV.5.4(ii)]), which states that the inequality $n+2 k \leq\left(d^{2}-4 d+6\right) / 2$ is sufficient for the non-emptiness of $V_{d}\left(n A_{1}, k A_{2}\right)$. In terms of $\delta=n+k$ and $\varkappa=2 n+3 k$, the latter inequality reads (16).

Remark 2.14 The non-emptiness condition (16) is asymptotically sharp for equiclassical families having expected dimension, since their codimension satisfies

$$
\varkappa-\delta \leq \operatorname{dim}\left|\mathcal{O}_{\mathbb{P}^{2}}(d)\right|=\frac{d(d+3)}{2}
$$

Theorem 2.15 Let $d \geq 3, \varkappa, \delta \geq 0$, and let $V_{d}^{\delta, \varkappa} \neq \emptyset$.

(a) If

$$
5 x-6 \delta \leq(d+3)^{2},
$$

then $V_{d}^{\delta, \varkappa}$ is locally regular and

$$
\operatorname{dim} V_{d}^{\delta, \varkappa}=\frac{d(d+3)}{2}-\varkappa+\delta .
$$

(b) If

$$
5 x-6 \delta \leq d^{2}+6 d-3,
$$

then a generic member of any component of $V_{d}^{\delta, \varkappa}$ is a curve with $3 \delta-\varkappa$ nodes and $x-2 \delta$ cusps as its only singularities.

(c) If

$$
\frac{11}{2} \varkappa+\frac{3}{2} \delta<d^{2}
$$

then $V_{d}^{\delta, \varkappa}$ is irreducible.

Remark 2.16 If a generic member of an irreducible component of $V_{d}^{\delta, \varkappa}$ is a curve with $n$ nodes and $k$ cusps, then inequality (17) reads $4 n+9 k \leq(d+3)^{3}$, the best known quadratic sufficient condition for the smoothness and expected dimension of the family of plane curves of degree $d$ with $n$ nodes and $k$ cusps [12, Corollary 4.4]. 
Proof of Theorem 2.15 (a) The LTI property is equivalent to the $h^{1}$-vanishing (7). Since $J^{\mathrm{ec}}\left(C, z_{i}\right) \supset J\left(C, z_{i}\right)$ for all $i=1, \ldots, r$, we can apply the $h^{1}$-vanishing criterion from [12, Proposition 4.1] sufficient for (7):

$$
\sum_{i=1}^{r} \gamma\left(C ; Z_{C, z_{i}}^{\mathrm{ec}}\right) \leq(d+3)^{3}
$$

where the $\gamma$-invariant can be computed as follows: for an irreducible zero-dimensional scheme $Z \subset C$ supported at a point $z \in \operatorname{Sing}(C)$ and such that its defining ideal satisfies $I_{Z} \supset J(C, z)$,

$$
\gamma(C ; Z)=\max \left\{4 \operatorname{deg} Z, \max _{D} \frac{(D \cdot C)_{z}^{2}}{(D \cdot C)_{z}-\operatorname{deg} Z}\right\},
$$

where $D$ runs over all plane curve germs defined by elements $g \in I_{Z}$ such that $(D \cdot C)_{z} \leq 2 \operatorname{deg} Z$, see [12, Formula (4.1)] and [15, Definition I.2.25]. Let us show that

$$
\gamma\left(C ; Z_{C, z_{i}}^{\mathrm{ec}}\right)=\frac{\varkappa\left(C, z_{i}\right)^{2}}{\delta\left(C, z_{i}\right)} .
$$

Indeed, by definition of $Z_{C, z_{i}}^{\mathrm{ec}}$, always $(D \cdot C)_{z} \geq \varkappa\left(C, z_{i}\right)$, and hence the maximal value of the right-hand side of $(21)$ is achieved for $(D \cdot C)_{z}=\varkappa\left(C, z_{i}\right)$, which together with $\operatorname{deg} Z_{C, z_{i}}^{\text {ec }}=\varkappa\left(C, z_{i}\right)-\delta\left(C, z_{i}\right)$ yields (22). Observing that $a^{2} / b \leq 5 a-6 b$ as long as $0<2 b \leq a \leq 3 b$, we derive the sufficient condition (17) for (20).

(b) Let $V$ be an irreducible component of $V_{d}^{\delta, \varkappa}, C \in V$ its generic member having singular points $z_{1}, \ldots, z_{r}$ of topological types $S_{1}, \ldots, S_{r}$, resp. Then the germ of $V$ at $C$ is the germ at $C$ of an equisingular family $V_{d}\left(S_{1}, \ldots, S_{r}\right)$. Suppose that $z_{1}$ is not a node or a cusp. By [30, pp. 144,158,164], see also [9, Theorem 2.25], the equisingular stratum $\mathrm{ES}_{1} \subset \mathrm{B}_{1}$ is smooth and its tangent space is $J^{\mathrm{es}}\left(C, z_{1}\right) / J\left(C, z_{1}\right)$, where $J^{\text {es }}\left(C, z_{1}\right) \subset \mathcal{O}_{\mathbb{P}^{2}, z_{1}}$ is the equisingular ideal. Furthermore, by [9, Proposition 5.10 and Theorem 5.12], $J^{\mathrm{ec}}\left(C, z_{1}\right) \subsetneq J^{\mathrm{es}}\left(C, z_{1}\right)$. Then there exists an intermediate ideal $J^{\mathrm{ec}}\left(C, z_{1}\right) \subsetneq J \subset J^{\mathrm{es}}\left(C, z_{1}\right)$ such that $\operatorname{dim} J / J^{\mathrm{ec}}\left(C, z_{1}\right)=1$. Introduce the zerodimensional scheme $Z^{\prime}=Z_{1} \cup \bigcup_{i \geq 2} Z_{C, z_{i}}^{\text {ec }}$ with $Z_{1}$ defined at $z_{1}$ by the ideal $J$. Then

$$
\operatorname{dim}_{C} V \leq h^{0}\left(\mathbb{P}^{2}, \mathcal{J}_{Z^{\prime}}(d)\right)-1
$$

Using (18), we will prove that

$$
H^{1}\left(\mathbb{P}^{2}, \partial_{Z^{\prime}}(d)\right)=0,
$$

which in turn will imply

$$
\begin{aligned}
\operatorname{dim}_{C} V & \leq h^{0}\left(\mathbb{P}^{2}, \partial_{Z^{\prime}}(d)\right)-1=\frac{d(d+3)}{2}-\operatorname{deg} Z^{\prime} \\
& =\frac{d(d+3)}{2}-\operatorname{deg} Z^{\mathrm{ec}}(C)-1=\frac{d(d+3)}{2}-\varkappa+\delta-1,
\end{aligned}
$$


a contradiction to claim (a). To establish (23), we again apply [12, Proposition 4.1] and verify the inequality

$$
\gamma\left(C ; Z_{1}\right)+\sum_{i=2}^{r} \gamma\left(C ; Z_{C, z_{i}}^{\mathrm{ec}}\right) \leq(d+3)^{2} .
$$

Similarly to the above computation of $\gamma\left(Z_{C, z_{i}}^{\mathrm{ec}}\right)$, we obtain

$$
\gamma\left(C ; Z_{1}\right) \leq \frac{\varkappa\left(C, z_{1}\right)^{2}}{\varkappa\left(C, z_{1}\right)-\operatorname{deg} Z_{1}}=\frac{\varkappa\left(C, z_{1}\right)^{2}}{\delta\left(C, z_{1}\right)-1} .
$$

It is an easy exercise to show (using formulas (5)) that $\delta\left(C, z_{1}\right) \geq 2$ and $\varkappa\left(C, z_{1}\right) \leq$ $5 \delta\left(C, z_{1}\right) / 2$, which yields

$$
\frac{\varkappa\left(C, z_{1}\right)^{2}}{\delta\left(C, z_{1}\right)-1} \leq 5 \varkappa\left(C, z_{1}\right)-6 \delta\left(C, z_{1}\right)+12 .
$$

Joining this with the inequality $\varkappa\left(C, z_{i}\right)^{2} / \delta\left(C, z_{i}\right) \leq 5 \varkappa\left(C, z_{i}\right)-6 \delta\left(C, z_{i}\right)$ obtained in step (a), we see that (18) implies (24), and hence (23), completing the proof of claim (b).

(c) Observe that (19) yields (18). Hence the irreducibility of $V_{d}^{\delta, \varkappa}$ will follow from the irreducibility of the family of plane curves of degree $d$ with $n=3 \delta-\varkappa$ nodes and $k=\varkappa-2 \delta$ cusps. By [12, Theorem 2], $V_{d}\left(n A_{1}, k A_{2}\right)$ is irreducible when $25 n / 2+$ $18 k<d^{2}$, which is equivalent to (19).

To apply duality isomorphisms (1), we go back to the parameters $d, g, c$.

Theorem 2.17 (a) The family $V_{d, g, c}$ is non-empty if $g \leq(d-1)(d-2) / 2$ and either

$$
c \geq g+\frac{5 d-8}{2}, \quad \text { or } \quad d \geq g+\frac{5 c-8}{2} .
$$

(b) Let $V_{d, g, c} \neq \emptyset$. Then

(b $V_{d, g, c}$ is locally regular if either

$$
5 c \geq 6 g+d^{2}-2 d-15, \quad \text { or } \quad 5 d \geq 6 g+c^{2}-2 c-15
$$

$\left(\mathrm{b}_{2}\right) V_{d, g, c}$ is irreducible if either

$$
11 c+3 g>\frac{21 d^{2}-31 d+6}{2}, \quad \text { or } \quad 11 d+3 g>\frac{21 c^{2}-31 c+6}{2} .
$$

Here each of the "either" inequality is a translation of the corresponding condition in Theorem 2.15, and each of the "or" inequality is the dual. We notice that the nodalcuspidal property is a priori not preserved by the duality. 
Example 2.18 We again illustrate the novelty of the dual criteria. Consider the family $V_{d}\left(k A_{2}\right)$ with large $d$ and $k=\left[(d+3)^{2} / 9\right]$. It is non-empty by Theorem $2.13(\mathrm{~b})$ and is LTI of expected dimension by Theorem 2.15 (a) (cf. Remark 2.16). The dual curves have degree and genus

$$
d^{\vee}=c=d(d-1)-3 k \sim \frac{2}{3} d^{2}, \quad g=\frac{(d-1)(d-2)}{2}-k \sim \frac{7}{18} d^{2},
$$

which correspond to the following numbers of virtual nodes and cusps

$$
n^{\vee} \sim \frac{2}{9} d^{4}, \quad k^{\vee} \sim \frac{19}{9} d^{2}
$$

but these values do not satisfy the other known LTI/expected dimension conditions: neither $k^{\vee}<3 d^{\vee}$, nor $4 n^{\vee}+9 k^{\vee}<\left(d^{\vee}+3\right)^{2}$.

\section{Duality of spacial curves and associated maps}

Duality for non-degenerate curves in $\mathbb{P}^{n}, n \geq 3$, was discovered by Piene [24, Section 5]. It differs from the general duality between subvarieties of $\mathbb{P}^{n}$, and it always takes curve to curve. It, furthermore, can be extended to curves in Grassmannians [23]. Relations between projective curves and curves in Grassmannians have been treated in [17]. We present here some basic material from [16,17,23,24] on spacial and associated curves, on their duality, and on associated maps between families of curves in projective spaces and Grassmannians. For the reader's convenience we provide elementary proofs, which can be traced back to Weyl [31] and which require only an undergraduate calculus. Finally, we formulate few problems on the geometry of equiclassical families of spacial curves and maps between them.

Associated and dual curves. We consider curves in the (complex) projective spaces $\mathbb{P}^{n}, n \geq 2$, as maps of Riemann surfaces to $\mathbb{P}^{n}$. Let $\mathcal{M}_{g, 0}\left(\mathbb{P}^{n}, d\right), d \geq 1$, be the moduli space of stable maps $f: S \rightarrow \mathbb{P}^{n}$ of Riemann surfaces $S$ of genus $g \geq 0$ such that $\left[v_{*}(S)\right]=d\left[\mathbb{P}^{1}\right] \in H_{2}\left(\mathbb{P}^{n}\right)$. It is a quasiprojective variety, see, for instance, [10, Theorem 1]. Denote by $\mathcal{M}_{g, 0}^{\text {nd }}\left(\mathbb{P}^{n}, d\right) \subset \mathcal{M}_{g, 0}\left(\mathbb{P}^{n}, d\right)$ the subspace of non-degenerate curves, that is (isomorphism classes of) maps $f: S \rightarrow \mathbb{P}^{n}$ birational onto the image $f(S)$, which is not contained in any hyperplane in $\mathbb{P}^{n}$.

Let a curve $f: S \rightarrow \mathbb{P}^{n}, n \geq 2$, be non-degenerate. Let $s \in S$, and $t$ a regular parameter in the analytic germ $(S, s)$. In suitable projective coordinates $X=\left(x_{0}, \ldots, x_{n}\right)$, the map $f:(S, s) \rightarrow \mathbb{P}^{n}$ is given by a vector-function $X(t)=\left(x_{0}(t), \ldots, x_{n}(t)\right)$, cf. [16, p. 266], where

$$
\begin{gathered}
x_{0}(t)=1+O(t), \quad x_{1}(t)=t^{1+\beta_{0}}(1+O(t)), \quad \ldots, \\
x_{n}(t)=t^{n+\beta_{0}+\ldots+\beta_{n-1}}(1+O(t))
\end{gathered}
$$

Given $k=0, \ldots, n-1$, the number $\beta_{k}=\beta_{k}(f, s) \geq 0$ in the parametrization (25) does not depend on the choice of a local parameter and is called the $k$-th ramification 
index of $f$ at the point $s \in S$; the number $\beta_{k}(C)=\sum_{s \in S} \beta_{k}(f, s)$ is finite and is called the total $k$-th ramification index of the curve $C$.

A non-degenerate curve $C=\left[f: S \rightarrow \mathbb{P}^{n}\right]$ induces a well-defined associated curve $C_{k}=\left[f_{k}: S \rightarrow \operatorname{Gr}(k+1, n+1)\right]$, which parameterizes osculating $k$-planes to $C=C_{0}$, for all $k=1, \ldots, n-1$ (see details, for instance, in [16, Chapter 2, Section 4]). In the chosen coordinates (25), $f_{k}$ is locally given by the multivectorfunction $X(t) \wedge X^{\prime}(t) \wedge \cdots \wedge X^{(k)}(t)$. Denote $C_{k}=\mathrm{As}_{k}(C), k=0,1, \ldots, n-1$, where $C_{0}=C$. Observe that $C_{n-1}=\operatorname{As}_{n-1}(C)$ is a curve in the dual projective space $\left(\mathbb{P}^{n}\right)^{*}=\operatorname{Gr}\left(n, \mathbb{C}^{n+1}\right)$. Denote by $\left(\mathbb{C}^{n+1}\right)^{*}$ the space dual to $\mathbb{C}^{n+1}$. We have the following canonical isomorphisms for all $k=1, \ldots, n$ :

$$
\begin{aligned}
& *_{k}: \operatorname{Gr}\left(k, \mathbb{C}^{n+1}\right) \rightarrow \operatorname{Gr}\left(n+1-k,\left(\mathbb{C}^{n+1}\right)^{*}\right), \\
& *_{k}: \operatorname{Gr}\left(k,\left(\mathbb{C}^{n+1}\right)^{*}\right) \rightarrow \operatorname{Gr}\left(n+1-k,\left(\left(\mathbb{C}^{n+1}\right)^{*}\right)^{*}\right)=\operatorname{Gr}\left(n+1-k, \mathbb{C}^{n+1}\right),
\end{aligned}
$$

which send a linear subspace to the space of linear functionals vanishing on it. Clearly, $*_{k} *_{n+1-k}=$ Id for each $k=1, \ldots, n$.

Definition 3.1 Let $C^{\prime}=\left[f^{\prime}: S \rightarrow \mathbb{P}^{n}\right]$ and $C^{\prime \prime}=\left[f^{\prime \prime}: S \rightarrow\left(\mathbb{P}^{n}\right)^{*}\right]$ be two nondegenerate curves. We say that $C^{\prime \prime}$ is $k$-dual to $C^{\prime}$ (resp., $C^{\prime}$ is $(n-k)$-dual to $C^{\prime \prime}$ ) if $*_{k}\left(C_{k-1}^{\prime}\right)=C_{n-1-k}^{\prime \prime}$, and we say that $C^{\prime}$ and $C^{\prime \prime}$ are dual if $C^{\prime \prime}$ is $k$-dual to $C^{\prime}$ for each $k=1, \ldots, n-1$.

Obviously, if $C^{\prime \prime}$ is $k$-dual to $C^{\prime}$ for each $k=1, \ldots, n-1$ then $C^{\prime}$ is also $k$-dual to $C^{\prime \prime}$ for each $k=1, \ldots, n-1$. The key fact is the following duality statement.

Theorem 3.2 ([24, Theorem 5.1]) Let $C$ be a non-degenerate curve in $\mathbb{P}^{n}, n \geq 2$. Then $C^{*}=\operatorname{As}_{n-1}(C)$ is non-degenerate as well, and, moreover, $C$ and $C^{*}$ are dual curves. Furthermore, $\beta_{k}\left(C^{*}\right)=\beta_{n-1-k}(C)$ for all $k=0, \ldots, n-1$.

Proof Let $C$ be locally given by a vector-function $X(t)$ in the form (25). Recall that (see [16, pp. 263-264]) for a non-degenerate curve $C$, at all but finitely many points $z \in S$, the vectors $X, X^{\prime}, \ldots, X^{(n-1)}$ (computed in the parametrization (25)) are linearly independent, and $\beta_{k}(f, s)=0$ for all $k=0, \ldots, n-1$.

Denote $f^{*}=f_{n-1}$. The map $f^{*}:(S, s) \rightarrow\left(\mathbb{P}^{n}\right)^{*}$ is given by the $n \times n$-minors $y_{0}(t), \ldots, y_{n}(t)$ of the matrix formed by the rows $X, X^{\prime}, \ldots, X^{(n-1)}$. It is an easy exercise to show (after reducing the minimal common power of $t$ ) that

$$
\begin{aligned}
& y_{0}=t^{n+\beta_{n-1}+\cdots+\beta_{0}}\left(\lambda_{0}+O(t)\right), \quad y_{1}=t^{n-1+\beta_{n-1}+\cdots+\beta_{1}}\left(\lambda_{1}+O(t)\right), \quad \ldots, \\
& y_{k}=t^{n-k+\beta_{n-1}+\cdots+\beta_{k}}\left(\lambda_{k}+O(t)\right), \quad \ldots, \quad y_{n}=\lambda_{n}+O(t),
\end{aligned}
$$

where $\lambda_{0} \lambda_{1} \ldots \lambda_{n} \neq 0$. It follows that $C^{*}$ is non-degenerate,

$$
\beta_{k}\left(f^{*}, s\right)=\beta_{n-1-k}(f, s), \quad k=0, \ldots, n-1, \quad s \in S,
$$

and for a regular point $s \in S$, the map $f^{*}:(S, s) \rightarrow\left(\mathbb{P}^{n}\right)^{*}$ can also be recovered as the unique (up to proportionality) solution $Y=\left(y_{0}, \ldots, y_{n}\right)$ to the system

$$
X Y=0=X^{\prime} Y=\cdots=X^{(k)} Y=\cdots=X^{(n-1)} Y=0,
$$


where the product of vectors is understood as the sum of products of the corresponding coordinates. Differentiating equations (26) and combining them to each other, we obtain that

$$
X^{(k)} Y^{(l)}=0 \quad \text { for all } k, l \geq 0, \quad k+l \leq n-1 .
$$

In particular,

$$
X Y=X Y^{\prime}=\cdots=X Y^{(k)}=\cdots=X Y^{(n-1)}=0,
$$

which means that $C=\operatorname{As}_{n-1}\left(C^{*}\right)$.

Furthermore, if $n \geq 3$, it follows from (27) that, for each $k=1, \ldots, n-2$, we have

$$
X^{(i)} Y^{(j)}=0 \quad \text { for all } \quad 0 \leq i \leq k, \quad 0 \leq j \leq n-1-k .
$$

The vectors $Y, Y^{\prime}, \ldots, Y^{(n-1)}$ are linearly independent too, since $\mathrm{As}_{n-1}(C)$ is a non-degenerate curve. The established independence and equalities (28) say that $*_{k} \operatorname{As}_{k}(C)=\operatorname{As}_{n-1-k}\left(C^{*}\right)$ for all $k=1, \ldots, n-2$.

Plücker formulas. The degrees of the associated curves $d_{k}=\operatorname{deg} C_{k}, 1 \leq k \leq n-1$, can be defined by the Plücker embedding Gr $\left(k+1, \mathbb{C}^{n+1}\right) \rightarrow \Lambda^{k+1} \mathbb{C}^{n+1}$ or by intersection with a generic Schubert cell $W_{1}=W_{1}\left(L_{n-k-1}\right) \subset \operatorname{Gr}\left(k+1, \mathbb{C}^{n+1}\right)$ formed by the $(k+1)$-subspaces intersecting with a given $(n-k-1)$-subspace $L_{n-k-1}$, cf. [16, p. 268]. They can be computed from $d=\operatorname{deg} C, g$, and the ramification indices $\beta_{k}=\beta_{k}(C), k=0, \ldots, n-1$, via the Plücker formulas, see, for example, [16, p. 270] or [24, Section 3]:

$$
d_{k-1}-2 d_{k}+d_{k+1}=2 g-2-\beta_{k}, \quad k=0, \ldots, n-1,
$$

where $d_{-1}=d_{n}=0, d_{0}=d=\operatorname{deg} C$. In particular,

$$
\begin{aligned}
d & =n(1-g)+\frac{1}{n+1} \sum_{i=0}^{n-1}(n-i) \beta_{i}, \\
d^{*} & =n(1-g)+\frac{1}{n+1} \sum_{i=0}^{n-1}(n-i) \beta_{n-1-i}, \\
d+d^{*} & =\sum_{i=0}^{n-1} \beta_{i}-2 n(g-1), \quad \text { where } d^{*}=\operatorname{deg} C^{*}
\end{aligned}
$$

Associated maps. Let $\bar{d}=\left(d_{0}, \ldots, d_{n-1}\right) \in \mathbb{Z}_{>0}^{n}$. Introduce the space

$$
\mathcal{M}_{g, 0}^{\mathrm{nd}}\left(\mathbb{P}^{n}, \bar{d}\right)=\left\{C \in \mathcal{M}_{g, 0}\left(\mathbb{P}^{n}, d_{0}\right): \operatorname{deg} C_{k}=d_{k}, k=1, \ldots, n-1\right\}
$$

Theorem 3.3 Let $\bar{d} \in \mathbb{Z}_{>0}^{n}$ and $\mathcal{M}_{g, 0}^{\mathrm{nd}}\left(\mathbb{P}^{n}, \bar{d}\right) \neq \emptyset$. Then there are well-defined injective maps

$$
\mathrm{As}_{k}: \mathcal{M}_{g, 0}^{\mathrm{nd}}\left(\mathbb{P}^{n}, \bar{d}\right) \rightarrow \mathcal{M}_{g, 0}\left(\operatorname{Gr}\left(k+1, \mathbb{C}^{n+1}\right), d_{k}\right), \quad k=1, \ldots, n-1,
$$


which are birational onto their images. Furthermore,

$$
\mathrm{As}_{n-1}: \mathcal{M}_{g, 0}^{\mathrm{nd}}\left(\mathbb{P}^{n}, \bar{d}\right) \rightarrow \mathcal{M}_{g, 0}^{\mathrm{nd}}\left(\left(\mathbb{P}^{n}\right)^{*}, \bar{d}^{*}\right), \quad \bar{d}^{*}=\left(d_{n-1}, \ldots, d_{0}\right)
$$

is a birational homeomorphism, satisfying $\mathrm{As}_{n-1}^{2}=\mathrm{Id}$.

Proof The statement on $\mathrm{As}_{n-1}$ follows from Theorem 3.2. Suppose that $1 \leq k<n-1$. In fact, we need to show only the injectivity of $\mathrm{As}_{k}$. Let $C=\left[f: S \rightarrow \mathbb{P}^{n}\right]$ and $C^{\prime}=\left[f^{\prime}: S^{\prime} \rightarrow \mathbb{P}^{n}\right]$ be two non-degenerate curves such that $\operatorname{As}_{k}(C)=\operatorname{As}_{k}\left(C^{\prime}\right)$. It is enough to show that if some germs $f:(S, s) \rightarrow \mathbb{P}^{n}, f^{\prime}:\left(S^{\prime}, s^{\prime}\right) \rightarrow \mathbb{P}^{n}$ admit parameterizations $X(t), Y(t), t \in(\mathbb{C}, 0)$, such that for each $t \in(\mathbb{C}, 0)$

$$
Y(t) \wedge Y^{\prime}(t) \wedge \cdots \wedge Y^{(k)}(t)=\mu(t) \cdot X(t) \wedge X^{\prime}(t) \wedge \cdots \wedge X^{(k)}(t),
$$

with some non-vanishing scalar function $\mu(t)$, then $Y(t)=\lambda(t) X(t)$, where $\lambda(t)$ is a scalar function. Without loss of generality, we can assume that $X(t)$ is given by (25) with $\beta_{0}=\cdots=\beta_{k-1}=0$, in particular, $X \wedge X^{\prime} \wedge \cdots \wedge X^{(k)}$ does not vanish for $t \in(\mathbb{C}, 0)$. Relation (30) yields that $Y, Y^{\prime}, \cdots, Y^{(k)} \in \operatorname{Span}\left\{X, X^{\prime}, \cdots, X^{(k)}\right\}$. Particularly,

$$
Y(t)=\lambda(t) X(t)+\lambda_{1}(t) X^{\prime}(t)+\cdots+\lambda_{k}(t) X^{(k)}(t) .
$$

It follows that $Y^{\prime}(t)$ is a linear combination of $X, X^{\prime}, \ldots, X^{(k)}$ plus $\lambda_{k}(t) X^{(k+1)}(t)$. However, since $C$ is non-degenerate and $k<n-1$, the vector $X^{(k+1)}$ is linearly independent of $X, X^{\prime}, \ldots, X^{(k)}$, and hence $\lambda_{k}(t) \equiv 0$. Considering subsequently $Y^{\prime \prime}, \ldots, Y^{(k)}$, in the same manner we deduce that $\lambda_{1}(t) \equiv \ldots \equiv \lambda_{k-1}(t) \equiv 0$. Hence, $Y=\lambda X$.

In fact, the maps inverse to $\mathrm{As}_{k}, 1 \leq k \leq n-2$, can be explicitly exhibited as follows. Let $f: S \rightarrow \mathbb{P}^{n}$ be a non-degenerate curve, $f_{k}: S \rightarrow \operatorname{Gr}\left(k+1, \mathbb{C}^{n+1}\right)$ its $k$-th associated curve, $1 \leq k \leq n-1$. Let $s \in S$ and $\Lambda=f_{k}(s) \in \operatorname{Gr}\left(k+1, \mathbb{C}^{n+1}\right)$. Given a function germ $F \in \mathcal{O}_{\mathrm{Gr}\left(k+1, \mathbb{C}^{n+1}\right), \Lambda}$ vanishing at $\Lambda$, we denote by $\operatorname{ord}_{s}\left(F \circ f_{k}\right)$ the order of zero of $F \circ f_{k}$ at $s$. For any proper linear subspace $L \subset T_{\Lambda} \operatorname{Gr}\left(k+1, \mathbb{C}^{n+1}\right)$, we define the intersection multiplicity $\left(\left.f_{k}\right|_{S, s} \cdot L\right)$ as

$$
\min _{F}\left\{\operatorname{ord}_{s}\left(F \circ f_{k}\right): F(\Lambda)=0,\left.D_{\Lambda} F\right|_{L}=0\right\}
$$

Recall also that there exists a canonical identification, see, e.g. [19, Lecture 16],

$$
T_{\Lambda} \operatorname{Gr}\left(k+1, \mathbb{C}^{n+1}\right) \simeq \operatorname{Hom}\left(\Lambda, \mathbb{C}^{n+1} / \Lambda\right)
$$

Denote by $\pi_{\Lambda}: \mathbb{C}^{n+1} \rightarrow \mathbb{C}^{n+1} / \Lambda$ the standard projection. 
Lemma 3.4 Fix $m=1, \ldots, n-k-1$.

(a) There exists a unique linear subspace $L \subset T_{\Lambda} \operatorname{Gr}\left(k+1, \mathbb{C}^{n+1}\right)$ of dimension $m$ for which the intersection multiplicity $\left(\left.f_{k}\right|_{S, s} \cdot L\right)$ attains its maximal value. We call this subspace the osculating $m$-space to the curve $f_{k}: S \rightarrow \operatorname{Gr}\left(k+1, \mathbb{C}^{n+1}\right)$ at the point $s$, and denote it by $L_{m}\left(f_{k}, s\right)$.

(b) Via the identification (31) the following holds: the projective subspace

$$
\mathbb{P}\left(\pi_{\Lambda}^{-1} \operatorname{Span}\left(\bigcup_{\Lambda^{\prime} \in L_{m}\left(f_{k}, s\right)} \operatorname{Im} \Lambda^{\prime}\right)\right) \subset \mathbb{P}^{n}
$$

coincides with the osculating $(k+m)$-plane to the curve $f: S \rightarrow \mathbb{P}^{n}$ at the point $s$.

Proof Consider the canonical parametrization (25) of the germ $f:(S, s) \rightarrow \mathbb{P}^{n}$. The corresponding parametrization $X(t) \wedge X^{\prime}(t) \wedge \cdots \wedge X^{(k)}(t)$ of the germ $f_{k}:(S, s) \rightarrow$ $\operatorname{Gr}\left(k+1, \mathbb{C}^{n+1}\right)$ via elementary transformations converts a family of $(k+1) \times(n+1)$ matrices $F_{k}(t)=\left(I_{k+1}, A(t)\right)$, where $I_{k+1}$ is the unit matrix of size $k+1$, and $A=\left(a_{i j}(t)\right)_{i=0, \ldots, k, j=1, \ldots, n-k}$ is a $(k+1) \times(n-k)$ matrix, in which

$$
a_{i j}=t^{\gamma(i, j)}\left(a_{i j}^{(0)}+O(t)\right), \quad \gamma(i, j)=k+j-i+\sum_{i \leq r \leq k+j-1} \beta_{r},
$$

and for all $j=1, \ldots, n-k$,

$$
a_{k j}^{(0)}=\prod_{r=1}^{k}\left(k+2-r+\sum_{l=r}^{k+1} \beta_{l}\right) \cdot\left(\prod_{r=1}^{k}\left(k+1-r+\sum_{l=r}^{k} \beta_{l}\right)\right)^{-1} \neq 0 .
$$

Note that the coordinate system $\left(a_{i j}, i=0, \ldots, k, j=1, \ldots, n-k\right)$ in a neighborhood of $\Lambda$ in $\operatorname{Gr}\left(k+1, \mathbb{C}^{n+1}\right)$ comes from the projection $(O, A) \mapsto A$ onto $T_{\Lambda} \operatorname{Gr}\left(k+1, \mathbb{C}^{n+1}\right) \simeq \operatorname{Hom}\left(\Lambda, \mathbb{C}^{n+1} / \Lambda\right)$, cf. [19, Example 16.1].

We have

$$
\begin{aligned}
F_{k}(t)=\left(I_{k+1}, O\right) & +t^{1+\beta_{k}} \cdot\left(O, A_{1}^{(0)}\right)+\cdots \\
& +t^{n-k+\beta_{k}+\cdots+\beta_{n-1}} \cdot\left(O, A_{n-k}^{(0)}\right)+O\left(t^{\gamma}\right),
\end{aligned}
$$

where $\gamma>n-k+\beta_{k}+\cdots+\beta_{n-1}$, and $A_{r}^{(0)}$ contains the only non-zero diagonal $\left(a_{i j}^{(0)}\right)_{i-j=k-r}$ with at least one non-zero element $a_{k r}^{(0)}$. It follows that the maximal value of $\left(\left.f_{k}\right|_{S, s} \cdot L\right)$ over $m$-dimensional subspaces of $T_{\Lambda} \operatorname{Gr}\left(k+1, \mathbb{C}^{n+1}\right)$ equals $m+1+\beta_{k}+$ $\cdots+\beta_{k+m}$ and is attained only at the space $L$ spanned by the (linearly independent) elements $A_{1}^{(0)}, \ldots, A_{m}^{(0)}$. The space generated by the rows of these matrices lifts to $\mathbb{C}^{n+1}$ as the span of the first $k+m+1$ unit vectors $e_{0}, \ldots, e_{k+m}$, and its projectivization is just the $(k+m)$-th osculating plane of the curve $f: S \rightarrow \mathbb{P}^{n}$ at $s \in S$.

As a direct consequence of Lemma 3.4 one obtains. 
Corollary 3.5 Let $n \geq 3$. For any $k=1, \ldots, n-2$ and $m=1, \ldots, n-k-1$, there exists a birational map

$$
\operatorname{As}_{m}^{k}: \operatorname{As}_{k}\left(\mathcal{M}_{g, 0}^{\mathrm{nd}}\left(\mathbb{P}^{n}, \bar{d}\right)\right) \rightarrow \operatorname{As}_{k+m}\left(\mathcal{M}_{g, 0}^{\mathrm{nd}}\left(\mathbb{P}^{n}, \bar{d}\right)\right)
$$

which is well-defined on a Zariski open, dense subset of $\operatorname{As}_{k}\left(\mathcal{M}_{g, 0}^{\mathrm{nd}}\left(\mathbb{P}^{n}, \bar{d}\right)\right)$ and which extends to a homeomorphism

$$
\widehat{\mathrm{As}}_{m}^{k}: \operatorname{As}_{k}\left(\mathcal{M}_{g, 0}^{\mathrm{nd}}\left(\mathbb{P}^{n}, \bar{d}\right)\right) \rightarrow \operatorname{As}_{k+m}\left(\mathcal{M}_{g, 0}^{\mathrm{nd}}\left(\mathbb{P}^{n}, \bar{d}\right)\right)
$$

Furthermore, the relations $\widehat{\mathrm{As}}_{m}^{k} \circ \mathrm{As}_{k}=\mathrm{As}_{k+m}$ and $\left(\mathrm{As}_{k}\right)^{-1}=* \widehat{\mathrm{As}}_{n-k-1}^{k}$ are satisfied.

Proof Let $f: S \rightarrow \mathbb{P}^{n}$ represent a smooth point of a component of $\mathcal{M}_{g, 0}^{\text {nd }}\left(\mathbb{P}^{n}, \bar{d}\right)$, and let $s \in S$ be a generic point. Then $\beta_{i}(f, s)=0, i=0, \ldots, n-1$, and the canonical parametrization (25) takes form

$$
x_{0}=1, \quad x_{1}=t(1+O(t)), \quad \ldots, \quad x_{n}=t^{n}(1+O(t)) .
$$

Then, in a smooth neighborhood $\mathcal{U} \subset \mathcal{M}_{g, 0}^{\text {nd }}\left(\mathbb{P}^{n}, \bar{d}\right)$ of $f: S \rightarrow \mathbb{P}^{n}$ we have a parametrization $u \in \mathcal{U} \mapsto\left[f_{u}: S_{u} \rightarrow \mathbb{P}^{n}\right]$, a smooth section $u \in \mathcal{U} \mapsto s(u) \in S_{u}$, and a universal parametrization $t \in(\mathbb{C}, 0) \rightarrow\left(S_{u}, s(u)\right)$ given by

$$
x_{0}(t)=1, \quad x_{1}(t)=t(\xi(u)+O(t)), \quad \ldots, \quad x_{n}=t^{n}\left(\xi_{n}(u)+O(t)\right),
$$

with $\xi_{1}, \ldots, \xi_{n}$ smoothly depending on $u \in \mathcal{U}$. Finally, the computations in the proof of Lemma 3.4 provide a smooth parametrization of a neighborhood of $f_{k}: S \rightarrow$ $\operatorname{Gr}\left(k+1, \mathbb{C}^{n+1}\right)$ in $\operatorname{As}_{k}\left(\mathcal{M}_{g, 0}^{\text {nd }}\left(\mathbb{P}^{n}, \bar{d}\right)\right)$ and define $\mathrm{As}_{m}^{k}$ as a smooth analytic map in that neighborhood. Combining this with Theorem 3.3, we obtain all statements.

Remark 3.6 (a) It follows from Corollary 3.5 that two curves $C^{\prime} \subset \mathbb{P}^{n}$ and $C^{\prime \prime} \subset$ $\left(\mathbb{P}^{n}\right)^{*}$ are dual if they are $k$-dual for at least one $k=1, \ldots, n-1$, see Definition 3.1.

(b) A birational homeomorphism may not be an isomorphism, what one can see in an elementary example of such a map between the line and a plane cuspidal cubic. To prove (or disprove) that the maps in Corollary 3.5 are isomorphisms, one should study the behavior of these maps at singularities of the spaces $\operatorname{As}_{k}\left(\mathcal{M}_{g, 0}^{\text {nd }}\left(\mathbb{P}^{n}, \bar{d}\right)\right)$.

Integrable curves in Grassmannians. The curves in Grassmannians, which are associated to non-degenerate projective curves, are called integrable curves. They admit the following characterization.

Given a linear subspace $V \subset \mathbb{C}^{n+1}$ such that $\operatorname{dim} V>k+1$, we obtain a natural embedding $\operatorname{Gr}(k+1, V) \hookrightarrow \operatorname{Gr}\left(k+1, \mathbb{C}^{n+1}\right)$, whose image is called a Grassmannian subvariety of $\operatorname{Gr}\left(k+1, \mathbb{C}^{n+1}\right)$. A curve $\varphi: S \rightarrow \operatorname{Gr}\left(k+1, \mathbb{C}^{n+1}\right)$ will be called $(k, n)$ non-degenerate ${ }^{2}$ if its image is not contained in any proper Grassmannian subvariety of $\operatorname{Gr}\left(k+1, \mathbb{C}^{n+1}\right)$.

\footnotetext{
${ }^{2}$ In this sense, a non-degenerate curve $\varphi: S \rightarrow \mathbb{P}^{n}$ is $(0, n)$-non-degenerate.
} 
Remark 3.7 Let us illustrate the difference between the non-degeneracy of curves in the projective space and the $(k, n)$-non-degeneracy of curves in Grassmannians. Consider, for example, a cubic normal curve $C=\left[f: \mathbb{P}^{1} \rightarrow \mathbb{P}^{3}\right]$. Its first associated curve $f_{1}: \mathbb{P}^{1} \rightarrow \operatorname{Gr}\left(2, \mathbb{C}^{4}\right)$ is $(1,3)$-non-degenerate, cf. Theorem 3.8 below, but it becomes degenerate after the Plücker embedding $\operatorname{Gr}\left(2, \mathbb{C}^{4}\right) \hookrightarrow \mathbb{P}^{5}$. Indeed, choosing the parametrization $x_{0}=1, x_{1}=t, x_{2}=t^{2}, x_{3}=t^{3}$ for $C$, we obtain the following Plücker coordinates of its first associated curve embedded into $\mathbb{P}^{5}: \pi_{03}=3 t^{2}$ and $\pi_{12}=t^{2}$, which means that the spoken curve lies in the hyperplane $\pi_{03}-3 \pi_{12}=0$.

Let $t \in(\mathbb{C}, 0) \mapsto L(t)=\operatorname{Span}\left\{X_{0}(t), \ldots, X_{k}(t)\right\} \subset \mathbb{C}^{n+1}$ be a parametrization of a curve $\varphi: S \rightarrow \operatorname{Gr}\left(k+1, \mathbb{C}^{n+1}\right)$ in a neighborhood of a point $s \in S$. Denote $L^{\prime}(t)=\operatorname{Span}\left\{X_{0}^{\prime}(t), \ldots, X_{k}^{\prime}(t)\right\}$ and let $\widehat{\varphi}(s)=L(0)+L^{\prime}(0)$. Clearly, the subspace $\widehat{\varphi}(s) \subset \mathbb{C}^{n+1}$ does not depend on the choice of a local parameter $t$ and a moving frame $\left(X_{0}(t), \ldots, X_{k}(t)\right)$.

Theorem 3.8 Let $n \geq 3$ and $1 \leq k \leq n-2$. A curve $\varphi: S \rightarrow \operatorname{Gr}\left(k+1, \mathbb{C}^{n+1}\right)$ is integrable if and only if it is $(k, n)$-non-degenerate, and $\operatorname{dim} \widehat{\varphi}(s)=k+2$ for almost all points $s \in S$.

Proof If $f: S \rightarrow \mathbb{P}^{n}$ is a non-degenerate curve, then its associated curve $f_{k}: S \rightarrow$ $\operatorname{Gr}\left(k+1, \mathbb{C}^{n+1}\right)$ is non-degenerate too. Furthermore, in parametrization (25), we have

$$
\operatorname{dim} \widehat{f_{k}}(s)=\operatorname{dim} \operatorname{Span}\left\{X(0), X^{\prime}(0), \ldots, X^{(k+1)}(0)\right\}=k+2,
$$

which holds for all points $s \in S$, where $\beta_{0}(f, s)=\ldots=\beta_{k}(f, s)=0$, i.e., for almost all points of $S$.

Now, let $\varphi: S \rightarrow \operatorname{Gr}\left(k+1, \mathbb{C}^{n+1}\right)$ be a non-degenerate curve with $\operatorname{dim} \widehat{\varphi}(s)=k+2$ for almost all points $s \in S$. We can choose a local parameter $t$ and a moving frame $\left(X_{0}(t), \ldots, X_{k}(t)\right)$ spanning $\varphi\left(s_{t}\right)$ in a neighborhood of a regular point $s_{0} \in S$ so that

$$
\widehat{\varphi}\left(s_{t}\right)=\operatorname{Span}\left\{X_{0}(t), \ldots, X_{k}(t), X_{0}^{\prime}(t)\right\}
$$

and correspondingly

$$
X_{1}^{\prime}(t), \ldots, X_{k}^{\prime}(t) \in \operatorname{Span}\left\{X_{0}(t), \ldots, X_{k}(t), X_{0}^{\prime}(t)\right\}
$$

Observe that

$$
X^{\prime \prime}(t) \notin \widehat{\varphi}\left(s_{t}\right) \quad \text { for almost all values } t \in(\mathbb{C}, 0) .
$$

Indeed, otherwise, we would immediately get that $X_{j}^{(i)}(0) \in \widehat{\varphi}\left(s_{0}\right)$ for all $j=0, \ldots, k$ and $i \geq 0$, and hence $\varphi(s) \subset \widehat{\varphi}\left(s_{0}\right) \subsetneq \mathbb{C}^{n+1}$ for all $s \in S$, contrary to the nondegeneracy assumption.

We claim that the curve $\varphi: S \rightarrow \operatorname{Gr}\left(k+1, \mathbb{C}^{n+1}\right)$ can be uniquely restored from the curve $\widehat{\varphi}: S \rightarrow \operatorname{Gr}\left(k+2, \mathbb{C}^{n+1}\right)$. Indeed, let $\psi: S \rightarrow \operatorname{Gr}\left(k+1, \mathbb{C}^{n+1}\right)$ be another curve such that $\widehat{\psi}=\widehat{\varphi}$, and let $\psi\left(s_{t}\right)=\operatorname{Span}\left\{Y_{0}(t), \ldots, Y_{k}(t)\right\}$ in a neighborhood of $s \in S$. Thus,

$$
Y_{i}(t)=a_{i}(t) X_{0}^{\prime}(t)+b_{i 0} X_{0}(t)+\cdots+b_{i k} X_{k}(t), \quad i=0, \ldots, k
$$


It follows that

$$
Y_{i}^{\prime}(t) \in a_{i}(t) X_{0}^{\prime \prime}(t)+\widehat{\varphi}\left(s_{t}\right)=a_{i}(t) X_{0}^{\prime \prime}(t)+\widehat{\psi}\left(s_{t}\right), \quad i=0, \ldots, k,
$$

which in view of (32) yields $a_{0}(t) \equiv \ldots \equiv a_{k}(t) \equiv 0$, and hence the required claim.

The statement of theorem follows now from the above claim and Corollary 3.5 by descending induction starting at $k=n-2$.

Open problems. It is natural to call the families $\mathcal{M}_{g, 0}^{\text {nd }}\left(\mathbb{P}^{n}, \bar{d}\right)$ defined by (29) the equiclassical families of curves in $\mathbb{P}^{n}, n \geq 2$. Similarly to the planar case, we formulate

Question 3.9 (a) Under what conditions is $\mathcal{M}_{g, 0}^{\mathrm{nd}}\left(\mathbb{P}^{n}, \bar{d}\right)$ equidimensional and what is $\operatorname{dim} \mathcal{M}_{g, 0}^{\text {nd }}\left(\mathbb{P}^{n}, \bar{d}\right)$ ? Under what conditions is $\mathcal{M}_{g, 0}^{\text {nd }}\left(\mathbb{P}^{n}, \bar{d}\right)$ irreducible? What are generic members of (the components of) $\mathcal{M}_{g, 0}^{\text {nd }}\left(\mathbb{P}^{n}, \bar{d}\right)$ ?

(b) Is the duality map $\operatorname{As}_{n-1}: \mathcal{M}_{g, 0}^{\text {nd }}\left(\mathbb{P}^{n}, \bar{d}\right) \rightarrow \mathcal{M}_{g, 0}^{\text {nd }}\left(\mathbb{P}^{n}, \bar{d}^{*}\right)$, where $\bar{d}^{*}=\left(d_{n-1}, \ldots\right.$, $\left.d_{0}\right)$, an isomorphism?

Canuto [4] has considered a problem related to the question on a generic member of $\mathcal{M}_{g, 0}^{\mathrm{nd}}\left(\mathbb{P}^{n}, \bar{d}\right)$ and proved that any compact Riemann surface, having a non-special linear system $g_{d}^{n}, n \geq 3$, without base points, can be mapped to $\mathbb{P}^{n}$ as a non-degenerate curve of degree $d$ so that its first $n-2$ associated curves are smooth.

Following the treatment of curves in Grassmannians in [4,23], one can state problems similar to those in Question 3.9. However, families of integrable curves

$$
\mathcal{M}_{g, 0}^{\mathrm{nd}}\left(\operatorname{Gr}\left(k+1, \mathbb{C}^{n+1}\right), \bar{d}\right)=\operatorname{As}_{k}\left(\mathcal{M}_{g, 0}^{\mathrm{nd}}\left(\mathbb{P}^{n}, \bar{d}\right)\right), \quad k=1, \ldots, n-2,
$$

seem to be most interesting.

Question 3.10 (a) Are the maps $\operatorname{As}_{k}: \mathcal{M}_{g, 0}^{\text {nd }}\left(\mathbb{P}^{n}, \bar{d}\right) \rightarrow \mathcal{M}_{g, 0}^{\mathrm{nd}}\left(\operatorname{Gr}\left(k+1, \mathbb{C}^{n+1}\right), \bar{d}\right)$, $k=1, \ldots, n-2$, isomorphisms? Are the maps $\widehat{\mathrm{As}}_{m}^{k}: \mathcal{M}_{g, 0}^{\mathrm{nd}}\left(\mathrm{Gr}\left(k+1, \mathbb{C}^{n+1}\right), \bar{d}\right) \rightarrow$ $\mathcal{M}_{g, 0}^{\mathrm{nd}}\left(\operatorname{Gr}\left(k+m+1, \mathbb{C}^{n+1}\right), \bar{d}\right), 1 \leq k \leq n-2,1 \leq m \leq n-k-1$, isomorphisms?

(b) What are generic members of (the components of) $\mathcal{M}_{g, 0}^{\text {nd }}\left(\operatorname{Gr}\left(k+1, \mathbb{C}^{n+1}\right), \bar{d}\right), k=$ $1, \ldots, n-2$ ?

Acknowledgments Main ideas behind this work have appeared during the visit of the second author to the Higher School of Economics (Moscow), and a part of this work has been done during the second author's visits to the Technische Universität Kaiserslautern and to the Centre Interfacultaire Bernoulli at EPFL, Lausanne. We are grateful to these institutions for support and excellent working conditions. We thank Gert-Martin Greuel for useful discussions and Ragni Piene for important remarks on the preliminary version of the paper and providing us with references on the subject.

\section{References}

1. Akyol, A., Degtyarev, A.: Geography of irreducible plane sextics (2014). arXiv:1406.1491

2. Arbarello, E., Cornalba, M.: A few remarks about the variety of irreducible plane curves of given degree and genus. Ann. Sci. École Norm. Sup. (4) 16(3), 467-488 (1983)

3. Calabri, A., Paccagnan, D., Stagnaro, E.: Plane algebraic curves with many cusps, with an appendix by Eugenii Shustin. Ann. Mat. Pura Appl. (4) 193(3), 909-921 (2014) 
4. Canuto, G.: Associated curves and Plücker formulas in Grassmannians. Invent. Math. 53(1), 77-90 (1979)

5. Dedieu, T., Sernesi, E.: Equigeneric and equisingular families of curves on surfaces (2014). arXiv: 1410.4221

6. Degtyarev, A.I.: Isotopic classification of complex plane projective curves of degree 5. Leningrad Math. J. 1(4), 881-904 (1990)

7. Degtyarev, A.: Topology of Algebraic Curves. De Gruyter Studies in Mathematics, vol. 44. Walter de Gruyter, Berlin (2012)

8. Diaz, S.: Irreducibility of the equiclassical locus. J. Differential Geom. 29(3), 489-498 (1989)

9. Diaz, S., Harris, J.: Ideals associated to deformations of singular plane curves. Trans. Amer. Math. Soc. 309(2), 433-468 (1988)

10. Fulton, W., Pandharipande, R.: Notes on stable maps and quantum cohomology. In: Kollár, J., Lazarsfeld, R., Morrison, D.R. (eds.) Algebraic Geometry Santa Cruz 1995. Proceedings of Symposia in Pure Mathematics, vol. 62, Part 2, pp. 45-96. American Mathematical Society, Providence (1997)

11. Greuel, G.-M., Karras, U.: Families of varieties with prescribed singularities. Compos. Math. 69(1), 83-110 (1989)

12. Greuel, G.-M., Lossen, C., Shustin, E.: Castelnuovo function, zero-dimensional schemes and singular plane curves. J. Algebraic Geom. 9(4), 663-710 (2000)

13. Greuel, G.-M., Lossen, C., Shustin, E.: Equisingular families of projective curves. In: Catanese, F., et al. (eds.) Global Aspects of Complex Geometry, pp. 171-209. Springer, Berlin (2006)

14. Greuel, G.-M., Lossen, C., Shustin, E.: Introduction to Singularities and Deformations. Springer Monographs in Mathematics. Springer, Berlin (2007)

15. Greuel, G.-M., Lossen, C., Shustin, E.: Singular Algebraic Curves. Monograph in preparation

16. Griffiths, P., Harris, J.: Principles of Algebraic Geometry. Wiley, New York (1978)

17. Griffiths, P., Harris, J.: Algebraic geometry and local differential geometry. Ann. Sci. École Norm. Sup. (4) 12(3), 355-452 (1979)

18. Harris, J.: On the Severi problem. Invent. Math. 84(3), 445-461 (1986)

19. Harris, J.: Algebraic Geometry. Graduate Texts in Mathematics, vol. 133. Springer, New York (1995)

20. Kang, P.-L.: On the variety of plane curves of degree $d$ with $\delta$ nodes and $\kappa$ cusps. Trans. Amer. Math. Soc. 316(1), 165-192 (1989)

21. Kang, P.-L.: A note on the variety of plane curves with nodes and cusps. Proc. Amer. Math. Soc. 106(2), 309-312 (1989)

22. Kulikov, Vik.S.: A remark on classical Pluecker's formulae (2011). arXiv:1101.5042

23. Perkinson, D.: Curves in Grassmannians. Trans. Amer. Math. Soc. 347(9), 3179-3246 (1995)

24. Piene, R.: Numerical characters of a curve in projective $n$-space. In: Holm, P. (ed.) Real and Complex Singularities (Oslo, 1976), pp. 475-495. Sijthoff and Noordhoff, Alphen aan den Rijn (1977)

25. Segre, B.: Dei sistemi lineari tangenti ad un qualunque sistema di forme. Atti Accad. Naz. Lincei. Rend. Cl. Sci. Fis. Mat. Natur. (5) 33(1), 152-185 (1924)

26. Segre, B.: Esistenza e dimensione di sistemi continui di curve piane algebriche con dati caratteri. Atti Accad. Naz. Lincei. Rend. Cl. Sci. Fis. Mat. Natur. (6) 10, 31-38 (1929)

27. Severi, F.: Vorlesungen über Algebraische Geometrie. Teubner, Leipzig (1921)

28. Shustin, E.: Real plane algebraic curves with prescribed singularities. Topology 32(4), 845-856 (1993)

29. Shustin, E.: Equiclassical deformation of plane algebraic curves. In: Brieskorn, E., Arnold, V.I., Greuel, G.-M., Steenbrink, J.H.M. (eds.) Singularities. Progress in Mathematics, vol. 162, pp. 195-204. Birkhäuser, Basel (1998)

30. Wahl, J.M.: Equisingular deformations of plane algebroid curves. Trans. Amer. Math Soc. 193, 143170 (1974)

31. Weyl, H.: Meromorphic Functions and Analytic Curves. Annals of Mathematics Studies, vol. 12. Princeton University Press, Princeton (1943)

32. Zariski, O.: Dimension-theoretic characterization of maximal irreducible algebraic systems of plane nodal curves of a given order $n$ and with a given number $d$ of nodes. Amer. J. Math. 104(1), 209-226 (1982) 